\title{
Domain-Specific Modelling of Micro Manufacturing Processes for the Design of Alternative Process Chains
}

\author{
Daniel Rippel $^{1} \quad$ Michael Lütjen $^{1} \quad$ Michael Freitag $^{1,2}$ \\ ${ }^{1}$ BIBA - Bremer Institut für Produktion und Logistik GmbH at the University of Bremen, Germany, \\ $\{r i p$, ltj\}@biba.uni-bremen. de \\ ${ }^{2}$ Faculty of Production Engineering, University of Bremen, Germany, fre@biba. uni-bremen. de
}

\begin{abstract}
In the context of an industrial production of micro components, the planning and configuration of process chains constitutes a major factor of success for the involved companies. Besides very small tolerances and high quality requirements, high production speeds have to be achieved. Moreover, so called size-effects introduce additional uncertainties to the planning process. While the modelling methodology "Micro Process Planning and Analysis" provides a series of tools and methods to achieve a detailed planning and configuration of process chains in micro manufacturing, the high level of detail requires a comparably large amount of manual work, as well as a broad knowledge about available processes. Moreover, several processes can be substituted to achieve specific forms and shapes, while providing their own advantages and disadvantages for the overall production system. This article describes an extension to the methodology, which enables an automatic selection of suitable processes using geometry focused annotations. While these annotations only add minor efforts to the modelling process, they can be used to automatically derive alternative process chains. Particularly for production systems offering a broad range of processes, this extension reduces the manual effort in modelling and evaluating alternative process chains.
\end{abstract}

Keywords: micro manufacturing, process planning, process configuration, geometry oriented process chain design

\section{Introduction}

During the last years the demand for metallic micro parts has increased continuously. While they become increasingly smaller, their shape's complexity and level of functional integration constantly increases (Wulfberg et al., 2010; Hansen et al., 2006; Mounier, Bonnabel, 2013). Aside from more complex applications for micro components, an increasing application of these components within the growth markets of medical- and consumer-electronics constitutes a primary driver for this development (Mounier, Bonnabel, 2013). Besides the growing demand for Micro-Electro-MechanicalSystems (MEMS), which are generally produced using methods from the semi-conductor industry, the demand for metallic micromechanical components increases similarly. These are generally used as connectors for MEMS, casings, or contacts. These micromechanical components are usually manufactured by applying processes from the areas of micro forming, micro injection, micro milling etc. (Hansen et al., 2006; Fu, Chan, 2012). Particularly, cold forming processes constitute a performant option for the realization of an economic mass production of metallic micromechanical components. These types of processes generally provide high throughput rates at comparably low energy and waste costs (DeGarmo et al., 2003).

An industrial production of such components is usually characterized by high throughput rates up to several hundred parts per minute (Flosky, Vollertsen, 2014), whereby very small tolerances have to be achieved. These tolerances result from the components' small dimensions, which are by definition smaller than one millimeter in at least two geometrical dimensions (Geiger et al., 2001). Moreover, so-called size-effects can result in increasing uncertainties and unexpected process behaviors when processes, originating from the macro domain, are scaled down to the micro level (Vollertsen, 2008). Additionally, micro manufacturing is an active and relatively young field of research for scientists as well as industrials, leading to a continuous development of new or enhanced processes and machines.

As a result, the planning and configuration of process chains constitutes one major success factor for an industrial production of metallic micromechanical components (Afazov, 2012). To cope with the occurrence of size-effects, companies require a highly precise planning not only of the single processes configurations, but also spanning the complete process chain. Thereby, interrelationships between processes, materials, tools and devices have to be considered. Small variations in single parameters can have significant influences along the process chain and can 
finally impede the compliance with the respective tolerances (Rippel et al., 2014).

This article describes an extension to the Micro Processes Planning and Analysis ( $\mu$-ProPlAn) methodology, which is designed to provide the necessary tools and procedures for an accurate planning and configuration of process chains within the micro domain (see e.g. (Rippel et al., 2014; Rippel et al., 2014b)). The methodology itself provides a set of methods to graphically model, plan and evaluate process chains across different levels of granularity. Nevertheless, due to the emergence of new technologies, there exist several possible processes to achieve the same product or work piece feature. For example, a hole can be placed within a metal sheet via drilling, laser-chemical or electrical ablation. The manual creation and evaluation of alternative process chain models can be time consuming and requires a broad knowledge of available technologies as well as of their different characteristics. In order to facilitate the search for the most economical solution, this article describes an extension to $\mu$-ProPlAn that allows an automatic selection of viable alternatives based on geometrical features of the desired work piece. The remainder of this section provides a short description of size-effects, followed by an overview of the state of the art in process planning within the micro domain. The next section gives a short introduction to the $\mu$-ProPlAn methodology and its components. Afterwards, the article describes the extension, its meta-model and a short example for its application. The article closes with a discussion of the extension as well as a description of planned future work on this topic.

\subsection{Size-Effects in Micro Cold Forming}

While cold forming processes are well established in mass production within macro manufacturing, scaling these processes down to the micro domain is only possible to a certain degree. With a decreasing scale of the machines, tools and work pieces, so called sizeeffects begin to emerge, which require changes and adaptations to the processes.

Vollertsen defines size effects as "deviations from intensive or proportional extrapolated extensive values of a process, which occur when scaling the geometrical dimensions" (Vollertsen, 2008). In this context, he defines intensive values as parameters, which are not expected to change due to a change of an object's mass (e.g. its temperature or its density) as well as extensive values that are expected to vary (e.g. the object's inertia force or its heat content). In general, size effects occur due to the inability to scale all relevant parameters equally (Vollertsen, 2008). For example, the downscaling of a metal sheet can result in stronger variations of its density due to local defects, although the density is considered an intensive variable. In macro manufacturing these variations can be ignored, while they can have drastic influences in micro manufacturing. Moreover, technical limitations can further facilitate the occurrence of size effects. For instance, the downscaling of mechanical grippers is limited by technical factors and only possible to a certain degree. For tiny work pieces, Van-der-Waals forces between the gripper and the work piece will eventually overcome the gravitational force at a certain point of miniaturization. As a result, the gripper will not be able to release the work piece without aid. Vollertsen defines three distinct categories of size effects (Vollertsen, 2008):

- Density size-effects occur, when the density of a material is held constant, while scaling down its geometrical dimensions. For instance, local defects become more serious with a continuing miniaturization. Thereby, the distribution of local defects within a material can lead to more delimited sets of good and bad parts.

- Shape size-effects occur due to the increasing ratio of an object's total surface area, compared to its volume. An example of this category is provided by the described imbalance of the adhesive force in relation to the gravitational force.

- Micro structure size-effects occur because micro structural features (e.g. the grain size or the surface roughness) cannot be scaled down the same way as the geometrical size of an object.

The occurrence of size-effects requires precise planning and configuration of all relevant technical parameters throughout a process chain. Due to size-effects and the continuous development of new processes and technologies for micro manufacturing, interrelations between those parameters can rarely be described comprehensively or are entirely unknown in several cases.

\subsection{Process Planning and Configuration in Micro Manufacturing}

Existing literature, describes only very few approaches that enable a joint planning of process chains as well as the technological and logistic configuration of the involved processes. During the last years, different articles focused on the configuration of specific processes (compare e.g. (Afazov, 2013)). Thereby, these approaches rely on detailed studies of the corresponding processes and are usually supported by highly detailed physical models in form of finite element simulations (e.g. (Afazov, 2012; Pietrzyk et al., 2008)). A different type of approach found in the literature focusses on the use of sample data (historical or experimental) as templates for the configuration of processes (e.g. (Sabotin et al., 2009)). Although both of these approaches allow for a precise configuration of single processes, the interrelations between different processes within a process chain cannot be considered easily. 
Moreover, the construction of finite element simulations as well as the direct application of historical information requires a comprehensive understanding of the processes and of the physical backgrounds, which in many cases is unavailable to the process planner due to size-effects or the novelty of processes.

In general, methods such as event-driven process chains, UML or simple flow charts are used in the context of process chain planning. While these methods do not enable a configuration of processes, Denkena et.al. proposed an approach that indirectly addresses this topic (Denkena et al., 2006). This approach relies on the modelling concept for process chains (Denkena, Tönshoff, 2011). In this concept, a process chain consists of different process elements, again consisting of operations. These operations are interconnected by so-called technological interfaces, which generally describe sets of pre- or post-conditions for each operation. While these interfaces can be configured manually, Denkena et al. extended this concept by proposing the use of physical, numerical or empirical models to estimate the relationships between the preand post-conditions (Denkena et al., 2006; Denkena et al., 2014). Although this approach enables configuration of the processes, the creation of these models requires a very detailed insight into the processes as stated before. Moreover, within the micro domain, a single model can easily be unsuitable to capture all relevant interrelations between process-, machine-, tool- and work pieceparameters, particularly under the influence of sizeeffects.

Based on the literature review, it can be concluded, that there is no method enabling a joint planning and configuration of process chains for the micro domain, which provides the necessary level of detail and generality to cope with size-effects as well as with the continuous development of new technologies in this domain.

\section{$2 \mu$-ProPIAn}

The modelling methodology $\mu$-ProPlAn covers all phases from the process and material flow planning to the configuration and evaluation of the processes and process chain models (Rippel et al., 2014). It enables an integrated planning of manufacturing, handling and quality inspection activities at different levels of detail, starting on the level of process chains, down to the level of cause-effect relations between single parameters. The methodology consists of a modelling concept, an accompanying procedure model as well as different methods for the evaluation of the modelled production systems' technological feasibility and logistic performance. All of these aspects are integrated in a software prototype.

The modelling concept consists of three views, which represent different levels of detail. The first view focusses on the top-level process chains, whereby the second view extends these process models by adding information on the specific material flow. The third view is used to further detail all relevant material flow elements (e.g. machines or work pieces) in terms of their parameters and the parameters' interrelations (causeeffect networks). Figure 1. depicts the most important entities used within the $\mu$-ProPlAn models and their hierarchical composition.



Figure 1. Major Entitties used within $\mu$-ProPlAn's models (Rippel et al., 2014).

The process view applies a notation closely conforming to the widely used notation of process chains, as described in the state of the art (Denkena, Tönshoff, 2011). In difference to the classical approach, process elements as well as operations are connected using extended process interfaces. These interfaces also include logistic parameters, asides from the technical parameters used within the classic approach. Additionally, operations act as interfaces to the next view: the material flow view.

On the one hand, the material flow view allows modeling of all material flow objects present in the modelled production scenario (i.e. machines/devices, work pieces, tools, operating supplies or workers). On the other hand, it is used to further detail operations by assigning those material flow objects that are used to conduct the operation. This enables modelling of specific production scenarios, for example, factories or production sites with specified resources as well as specific routes for each product (process chain). As a result, it becomes possible to evaluate the models using discrete-event material flow simulations regarding logistic aspects. For example, in case of an existing production system, $\mu$-ProPlAn can be used to assess the impact of new process chains (e.g. new products) on existing production plans or on the performance of the production system in general. Therefore, the $\mu$-ProPlAn software prototype can directly transform these models 


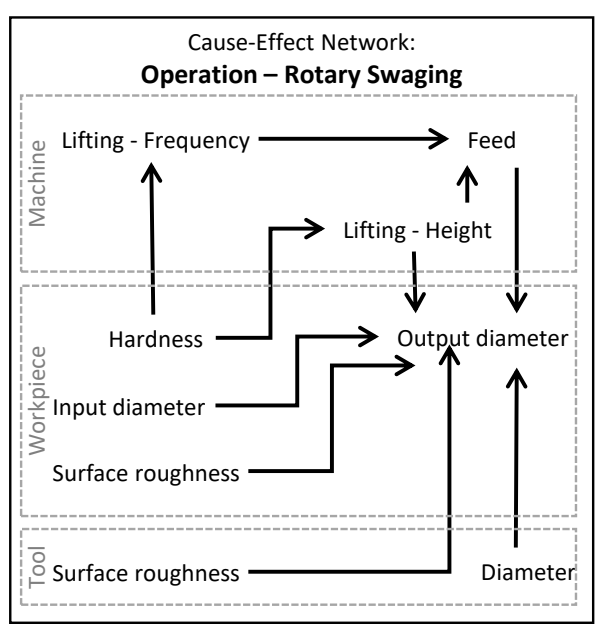

a)

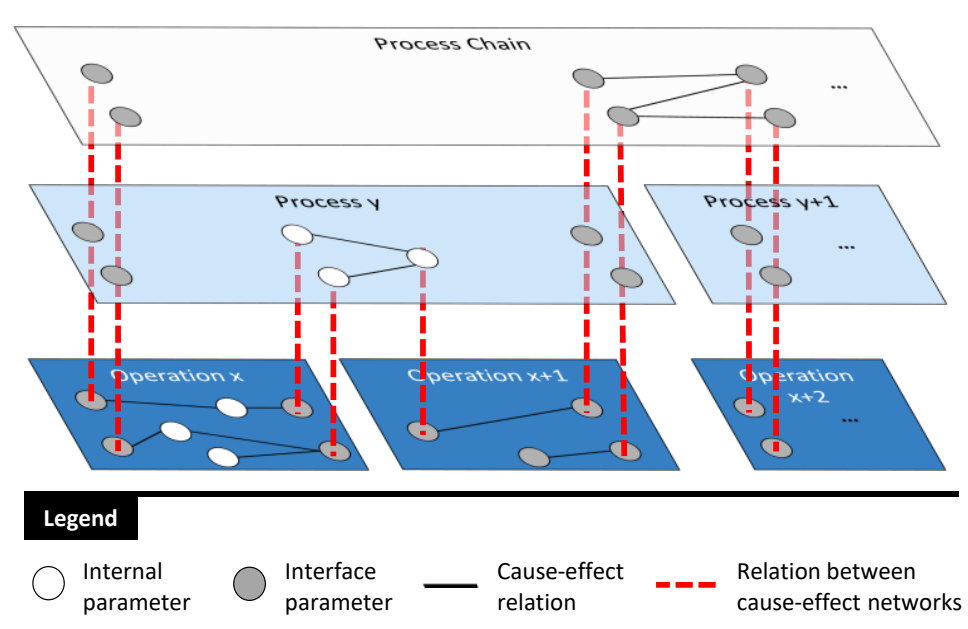

(b)

Figure 2. Hierarchy of cause-effect networks: (a) Exemplary composition of an operation from the networks of the corresponding machine, tool and work piece. (Rippel et al., 2014b); (b) Composition of higher level cause-effect-networks for process elements and process chains (Rippel et al., 2014).

into discrete-event simulation models for the jasima ${ }^{l}$ library and execute them. This allows an early detection of bottleneck machines or other undesired effects early during the planning stages. Consequently, this supports the selection of suitable resources, machines or devices for the new process chain.

The third view focusses on the configuration of the processes and process chains using cause-effect networks to describe the interrelationships between relevant process parameters. In contrast to holistic approaches like those described in the state of the art, each network consists of a set of parameters and a set of cause-effect relationships, forming a directed graph. The set of parameters consists of all technical and logistic characteristics that are relevant to describe the respective object's influence on the production process. In case of work pieces these are e.g. costs per unit, material properties or geometrical characteristics. For machines, these parameters include velocities, forces or other characteristics that can be set, calculated or measured (compare Figure 2.a for an example). From a modelling perspective, the cause-effect networks are modelled hierarchically. Each material flow object (work pieces, machines, tools, workers, etc.) holds its own cause-effect network or at least a set of describing parameters. When combining these single elements to operations, process elements or process chains, higher level cause-effect networks are created by introducing additional relationships between parameters of the networks or by connecting them through previously specified process interfaces (compare Figure 2.b).

The creation of cause-effect networks is divided into two steps: the qualitative modelling and the quantification. The quantitative model of the corresponding network is created by collecting all relevant parameters and denoting their influences among each other (cf. Figure 2.a). The second step concerns the quantification of the cause-effect networks. The objective is to enable the propagation of different parametrizations throughout the network. For example, this propagation allows estimating the process' outcome for different materials or machining strategies. In Figure 2.a, the use of a different material for the forging tool will result in a different surface roughness, thus influencing the overall process' output diameter. Through quantifying the cause-effect relationships, it is possible to estimate the results of parameter changes to all connected parameters along complete process chains.

In case of simple or well-known relations, $\mu$-ProPlAn allows to input mathematical formulas directly. Each formula thereby calculates a parameter's value based on its input parameters' values. For example, a continuous manufacturing process will have a duration according to the total length of the work piece divided by the selected feed velocity. More complex but well-established causeeffect relations could be included from literature, e.g. the calculation of the static friction of a tool and work piece based on their surface roughness. Nevertheless, in the area of micro manufacturing, different parameters can have a more significant impact than in the macro domain, resulting in the inclusion of parameters that can be neglected in macro manufacturing. In addition, size effects may induce a different behavior than usually observed. Therefore, it is often impossible to comprehensively describe all parameters and causeeffects relations directly. As a result, $\mu$-ProPlAn proposes the derivation of prediction models from experimental- or from production data. Therefore, the

\footnotetext{
${ }^{1}$ JAva SImulator for MAnufacturing and logistics: https://code.google.com/archive/p/jasima/
} 


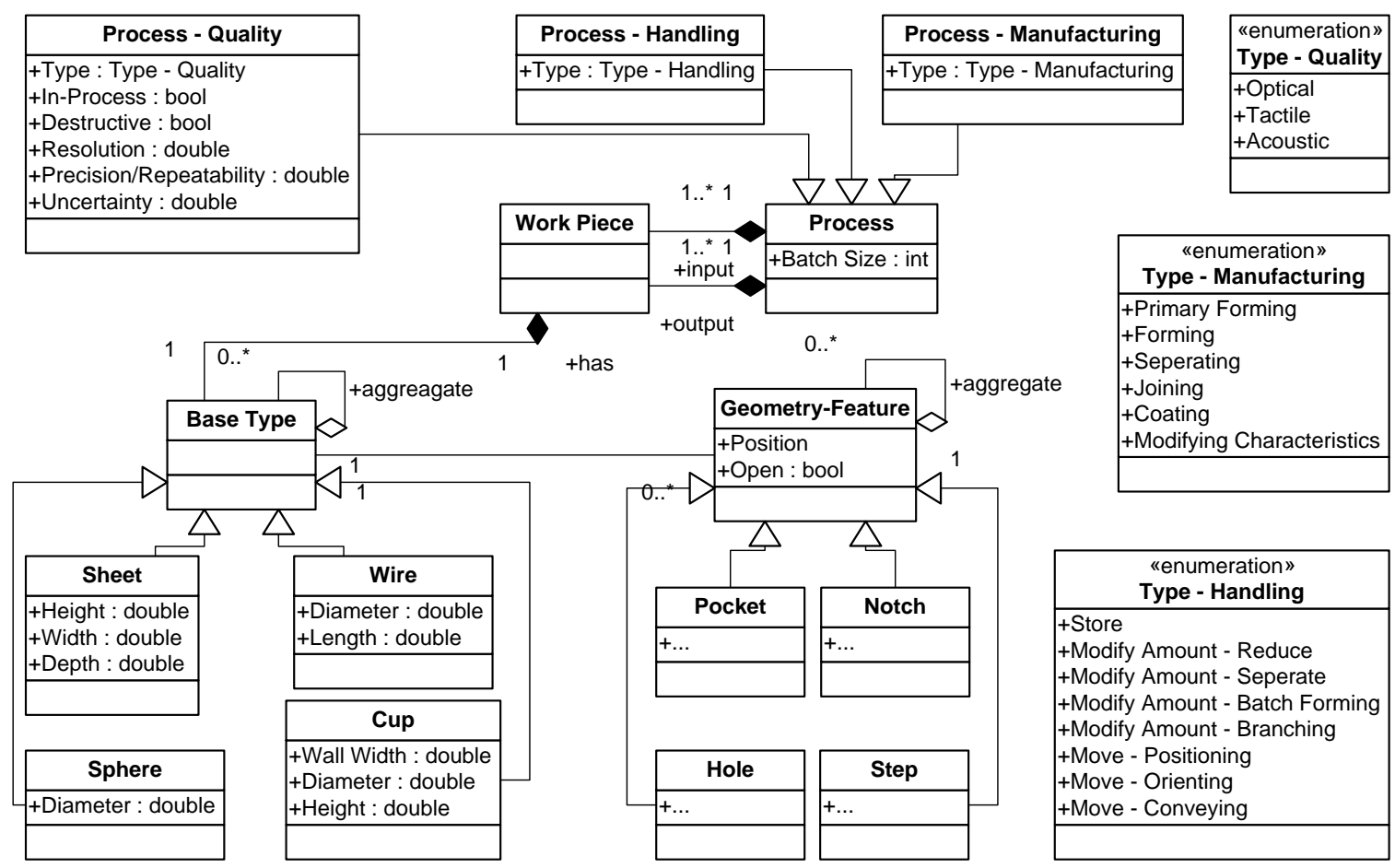

Figure 3. Simplified extension to the $\mu$-ProPlAn meta model using the UML notation. Attributes denote additional parameters for the respective cause-effect networks.

software prototype offers a variety of regression techniques from the area of Artificial Intelligence (e.g. Support-Vector Machines or Artificial Neural Networks) as well as statistical methods (e.g. the deduction of least square linear or polynomial regression models or locally weighted linear regression models). In practice, the application of locally weighted linear regression models has shown high accuracy in estimating unknown or hard-to-describe cause-effect relations (see e.g. (Rippel et al., 2014; Rippel et al., 2014b)). After quantification, each parameter contains a model (mathematical or prediction) that allows to estimate its value based on the remaining values in the cause-effect network.

As a result, these networks enable an in-depth evaluation of different process configurations (e.g. the use of different materials or different production velocities) and to assess the impacts of different choices on follow-up processes or the production system in general. As cause-effect networks and material flow elements are closely connected, $\mu$-ProPlAn can directly reflect changes to the configuration within the material flow simulation and evaluate these configurations e.g. regarding work-in-progress-levels, lead times or the products' estimated qualities.

While the methodology itself enables a highly precise planning of the process chains, as well as the configuration and evaluation of these chains, the high precision comes at the cost high modelling efforts. While the initial creation of material flow objects and their respective cause-effect networks can be conducted prior to the actual planning of a process chain, the introduction of new process chains (i.e. products) to an existing production system still requires a high amount of manual work. Thereby the production planner needs to select those processes and machines that result in the most economic overall production in terms of product quality as well manufacturing times and costs. In micro manufacturing, there exist a growing range of processes that can achieve similar results. As machines are often comparably small, production sites often house several different machines or processes, which leads to a possibly broad pool of alternative process chains. Nevertheless, each process provides its own characteristics in terms of velocity, cost, or other properties that can have a positive or a negative influence on the overall production system or on the products manufacturing process and characteristics. As the manual creation of alternative process chains can be quite time consuming and require a broad knowledge of available processes as well as their advantages and disadvantages, the next section describes an extension of the $\mu$-ProPlAn methodology that can be applied for an automatic selection and creation of process chain alternatives based on geometrical features of the desired product.

\section{Geometry oriented development of process chains}

The geometry oriented process chain design provides an alternative approach of modelling and designing process chains in $\mu$-ProPlAn. The current methodology assumes a manual modelling and evaluation of each alternative 
chain. The geometry-oriented approach focusses on additional annotations to the modelled process, in order to select and combine suitable processes. Thereby, each process is annotated with information on its capabilities and limitations. In addition, work pieces can be specified by their geometrical features. Using a constraint based search, suitable processes can be selected, combined and evaluated automatically. For these annotations, additional parameters are introduced to the cause-effect networks in order to describe which geometries can be achieved by a process to which extend as well as the respective pre-conditions. Basically, the $\mu$-ProPlAn meta model is adapted twofold: First, processes need to be annotated with their type (what they do). Second, each process requires annotation which geometrical features can be achieved under which circumstances.

The type of a process describes its function according to the standards DIN 8580 and VDI 2860. For manufacturing processes the type describes if it is a (primary) forming process, a separating or joining process, if it is a coating process or if it just modifies the work pieces characteristics without changing the geometry. For handling processes the type determines e.g. if it is a positioning or conveying process. Besides the definition of the type (optical, tactile, acoustic), quality inspection processes additionally require information if the process is destructive, if it can be used in-process as well as its resolution and uncertainties.

The second adaptation describes each process' preand post-conditions with respect to geometrical features. Thereby, each process gets annotated with a set of work pieces that can serve as input to the process as well as a set of work pieces which result from the process application to an input. In extension to the current meta model, each work piece has a base geometry, describing e.g. if the work piece is a sheet, a sphere, a wire etc.
Figure 3. lists some examples of base geometries with their respective parameters in the lower left part. This list can be extended for a given application or model in order to include additional base geometries as needed. Each base geometry can be combined with additional base geometries to compose more complex work pieces. Figure 4.a for example shows a combination of a wire with a cone shaped base geometry to represent a work piece that could act as a plunger for a micro valve. Each base geometry can be assigned zero or more geometrical features such as holes, steps, pockets or notches. Figure 4. lists some examples derived from the STEP-NC standard. While this standard focusses on the featurebased description of machining strategies, it offers comprehensive descriptions of geometrical features as well as their characteristics (parameters). To keep the image simple, these parameters have been omitted in Figure 4. as they can be directly taken from the STEPNC standard. Each of these features can again be assigned zero or more features on their own in a hierarchical manner. For example, this allows modelling of a block (Sheet) that has a pocket on its top side (Pocket), which again contains a hole (Hole) in its center.

By using these annotations, it becomes possible to apply constraint based search algorithms to select suitable processes. During the modelling process, the designer specifies the hierarchical order of base geometries and geometrical features required for the final product. The software tool can search through all stored processes within the model and select those, which can achieve each feature. In the example in Figure 4.b there is only one alternative to join a sphere to a wire (melting) but there exist two alternatives to form this sphere into a cone. As the characteristics of each base geometry and each feature are expressed as parameters integrated into the processes, cause-effect networks, the
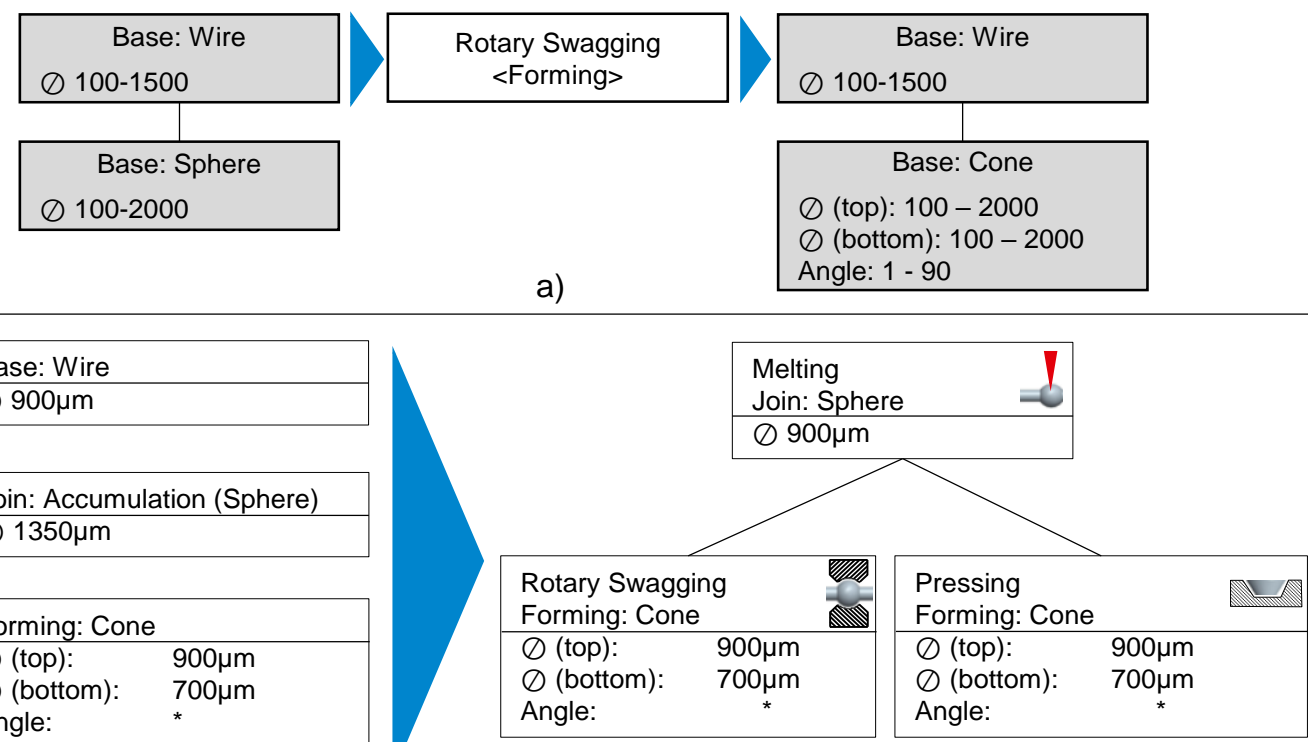

b)

Figure 4.(a) Examples for the geometry based annotations (b) Example for the automatic selection of processes 
configuration of each alternative process chain can be performed the usual way. As a result, technical characteristics (e.g. material or surface properties) can be estimated through the cause-effect networks, while logistic properties of different alternatives can be estimated by transforming and simulating the models using $\mu$-ProPlAns discrete-event material flow simulation.

\section{Discussion and Future Work}

Concerning the design and configuration of process chains in micro manufacturing, the selection of suitable machining processes and devices constitutes a major influential factor on the production's performance. Following the trends of desktop manufacturing as well as of the continuous development of new or improved processes for micro manufacturing, a broad range of alternate processes can be available. As a result, the manual creation and evaluation of alternative process chains can easily become a time consuming task, prone to errors. This article proposes an adaptation to the $\mu$ ProPlAn modelling methodology, focusing on the annotation of modelled processes, in order to enable an automatic derivation of alternative process chains, as well as their evaluation. This extension uses parts of commonly known standards like STEP-NC or different DIN and ISO standards to structure the required information and seamlessly integrate it with the causeeffect models used within $\mu$-ProPlAn.

The changes to the $\mu$-ProPlAns meta model provide a suitable approach to structure all the information required for an automatic selection of processes. As most of this information was already required in order to perform a configuration of the process chains in $\mu$ ProPlAn's current modelling approach (e.g. information which dimensions should be achieved with respect to the work pieces form), the annotation of the processes and work pieces only induces minor additional efforts. Moreover, the changes provide a better way to structure this information, which facilitates the models' consistency and thus renders them easier to maintain and extend. While the possibility to search for alternative processes is restricted by the number of stored processes, the use of additional databases could allow the use of process templates across several models. Future work will focus on the conceptualization and implementation of such a database as well as on the identification of a more general list of base geometries for micro manufacturing.

\section{Acknowledgements}

The authors gratefully acknowledge the financial support by Deutsche Forschungsgemeinschaft (DFG, German Research Foundation) for the Subproject C4 'Simultaneous Engineering' within the CRC 747 (Collaborative Research Center) "Mikrokaltumformen Prozesse, Charakterisierung, Optimierung".

\section{References}

S. M. Afazov, A. A. Becker, and T. H. Hyde. Development of a Finite Element Data Exchange System for chain simulation of manufacturing processes. In Advances in Engineering Software,47(1): 104 - 113, 2012.

S. M. Afazov. Modelling and simulation of manufacturing process chains. In CIRP Journal of Manufacturing Science and Technology, 6(1): 70 - 77, 2013.

E. P. DeGarmo, J. T. Black, and R. A. Kohser. Materials and Processes in Manufacturing, 9th ed.: Wiley, 2003.

B. Denkena, H. Rudzio, and A. Brandes. Methodology for Dimensioning Technological Interfaces of Manufacturing Process Chains. In CIRP Annals - Manufacturing Technology, 55(1): 497 - 500, 2006.

B. Denkena and H. K. Tönshoff. Prozessauslegung und integration in die Prozesskette. In Spanen - Grundlagen, B. Denkena and H. K. Tönshoff, Eds., Springer Verlag, Berlin, Heidelberg, pp. 339 - 362. 2011

B. Denkena, J. Schmidt, and M. Krüger. Data Mining Approach for Knowledge-based Process Planning. In Procedia Technology 15(1): 406 - 415, 2014.

H. Flosky and F. Vollertsen. Wear behaviour in a combined micro blanking and deep drawing process. In CIRP Annals - Manufacturing Technology 63(1): 281 - 284, 2014.

M. W. Fu and W. L. Chan. A review on the state-of-the-art microforming technologies. In The International Journal of Advanced Manufacturing Technology, 67(9): 2411 - 2437, 2012.

M. Geiger, M. Kleine, R. Eckstein, N. Tieslerl, and U. Engel. Microforming. In CIRP Annals - Manufacturing Technology, 50(2): 445-462, 2001.

H. N. Hansen, K. Carneiro, H. Haitjema, and L. De Chiffre. Dimensional Micro and Nano Technology. In Annals of the CIRP Annals, 55(2): 721-734, 2006.

E. Mounier and A. Bonnabel. Press Release Emerging MEMS, 29. August 2013. Yole Development, 2013.

M. Pietrzyk, L. Madej, and S. Weglarczyk. Tool for optimal design of manufacturing chain based on metal forming. In CIRP Annals - Manufacturing Technology, 57(1): 309 312, 2008.

D. Rippel, M. Lütjen, and B. Scholz-Reiter. A Framework for the Quality-Oriented Design of Micro Manufacturing Process Chains. In Journal of Manufacturing Technology Management, 25(7): 1028 - 1048, 2014.

D. Rippel, E. Moumi, M. Lütjen, B. Scholz-Reiter, and B. Kuhfuß. Application of Stochastic Regression for the Configuration of a Micro Rotary Swaging Process. In Mathematical Problems in Engineering, 2014b, http://dx.doi.org/10.1155/2014/360862.

I. Sabotin, J. Valentincic, M. Junkar, and A. Sluga. Process planning system for micro-products. In Proceedings of the 10th International Conference on Management of Innovative Technologies, p. 8, 2009.

F. Vollertsen. Categories of size effects. In Production Engineering, 2(4): 377-383, 2008.

J. P. Wulfsberg, T. Redlich, and P. Kohrs. Square Foot Manufacturing: a new production concept for micro manufacturing. In Production Engineering - Research and Development, 4(1): 75 - 83, 2010. 